ASC Report No. 13/2009

On the Suboptimality of the p-version Interior Penalty Discontinuous Galerkin Method

Emmanuil H. Georgoulis, Edward Hall, Jens Markus Melenk 


\section{Most recent ASC Reports}

02/2009 Samuel Ferraz-Leite, Jens Markus Melenk, Dirk Praetorius

Reduced Model in Thin-film Micromagnetics

01/2009 Markus Aurada, Samuel Ferraz-Leite, Dirk Praetorius

Convergence of Adaptive Boundary Element Methods

38/2008 Roberta Bosi

Classical Limit for Linear and Nonlinear Quantum Fokker-Planck Systems

37/2008 Federico Bassitti, Lucia Ladelli, Daniel Matthes

Central Limit Theorem for a Class of Ome-dimensional Equations

36/2008 Markus Brunk, Ansgar Jüngel

Self-Heating in a Coupled Thermo-Electric Circuit-Device Model

35/2008 Ansgar Jüngel, Daniel Matthes

Entropiemethoden für nichtlineare partielle Differentialgleichungen

34/2008 Ansgar Jüngel, Josipa-Pina Milišić

A Sixth-Order Nonlinear Parabolic Equation for Quantum Systems

33/2008 Ansgar Jüngel, Ingrid Violet

Mixed Entropy Estimates for the Porous-Medium Equation with Convection

31/2008 Markus Brunk, Ansgar Jüngel

Heating of Semiconductor Devices in Electric Circuits

31/2008 Anton Arnold, Matthias Ehrhardt, Maike Schulte, Ivan Sofronov

Discrete Transparent Boundary Conditions for the Schrödinger Equation on Circular Domains

Institute for Analysis and Scientific Computing

Vienna University of Technology

Wiedner Hauptstraße 8-10

1040 Wien, Austria

E-Mail: admin@asc.tuwien.ac.at

WWW: http://www.asc.tuwien.ac.at

FAX: $\quad+43-1-58801-10196$

ISBN 978-3-902627-02-5

(C) Alle Rechte vorbehalten. Nachdruck nur mit Genehmigung des Autors.

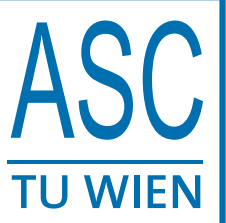




\title{
On the suboptimality of the $p$-version interior penalty discontinuous Galerkin method
}

\author{
Emmanuil H. Georgoulis;, Edward Hall ${ }^{\dagger} \&$ Jens Markus Melenk ${ }^{\ddagger}$
}

February 20, 2009

\begin{abstract}
We address the question of the rates of convergence of the $p$-version interior penalty discontinuous Galerkin method ( $p$-IPDG) for second order elliptic problems with non-homogeneous Dirichlet boundary conditions. It is known that the $p$-IPDG method admits slightly suboptimal a-priori bounds with respect to the polynomial degree (in the Hilbertian Sobolev space setting). An example for which the suboptimal rate of convergence with respect to the polynomial degree is both proven theoretically and validated in practice through numerical experiments is presented. Moreover, the performance of $p$ IPDG on the related problem of $p$-approximation of corner singularities is assessed both theoretically and numerically, witnessing an almost doubling of the convergence rate of the $p$-IPDG method.
\end{abstract}

\section{Introduction}

Discontinuous Galerkin (DG) methods for elliptic problems have gained popularity in recent years. Their great flexibility in the design of finite element methods make them good contenders in the area of $h p$ adaptive algorithms. Moreover, DG methods have shown to be accurate and stable numerical methods for the numerical approximation of convection-dominated convection-diffusion problems (see, e.g., [5] and the references therein). Historically, DG methods incorporate ideas from the classical Nitsche's method for the treatment of non-homogeneous Dirichlet boundary conditions [10] and from the penalty method [1].

To the best of our knowledge, the sharpest known general error bounds (in the Hilbertian Sobolev space setting) for the $h p$-version interior penalty DG method for second-order elliptic PDEs are due to Rivière, Wheeler and Girault [11] and Houston, Schwab and Süli [9]; when the error is measured in the (natural) energy norm, the a-priori bounds are optimal with respect to the meshsize $h$ but are suboptimal with respect to the polynomial degree $p$ by half an order of $p$.

Optimal error bounds for the $h p$-version interior penalty DG method are known in the case where the underlying discontinuous Galerkin finite element space admits an $H^{1}$-conforming subspace of the same polynomial order up to the boundary; see, e.g., [6, Theorem 8.2] and the subsequent discussion therein. In the case where the discontinuous Galerkin finite element space does not admit such an $H^{1}$-conforming subspace (e.g., when the mesh is highly irregular or when the Dirichlet boundary conditions are not represented exactly as traces of finite element functions), $h p$-optimal error bounds in the energy norm have been derived in [7] for the case of quadrilateral elements, provided the analytical solution admits additional regularity in the framework of augmented Sobolev spaces. Recently, in [13], a variation of the interior penalty DG method (which includes an additional penalization term resembling the local discontinuous Galerkin method) is proposed and $h p$-optimal error bounds are proven for the case of homogeneous Dirichlet boundary conditions.

\footnotetext{
*Department of Mathematics, University of Leicester, University Road, Leicester LE1 7RH, United Kingdom, e-mail: Emmanuil.Georgoulis@mcs.le.ac.uk

${ }^{\dagger}$ School of Mathematical Sciences, University of Nottingham, University Park, Nottingham, NG7 2RD, United Kingdom, e-mail: edward.hall@nottingham.ac.uk

${ }^{\ddagger}$ Institut für Analysis und Scientific Computing, Technische Universität Wien, Wiedner Hauptstrasse 8-10, A-1040 Wien, Austria, e-mail: melenk@tuwien.ac.at
} 
In this work, we focus on the $p$-version interior penalty discontinuous Galerkin finite element method ( $p$-IPDG), addressing the question of the rates of convergence for second order elliptic problems with non-homogeneous Dirichlet boundary conditions, when the underlying analytical solution belongs to a (standard) Hilbertian Sobolev space $H^{k}$. More specifically, we present an example for which the suboptimal rate of convergence with respect to the polynomial degree is both proven theoretically and validated through numerical experiments; hence, the known a-priori bounds from the literature $[11,9]$ are sharp, i.e., the $p$-IPDG method is indeed suboptimal by half an order of $p$.

Furthermore, we investigate the question of convergence rates for the $p$-IPDG in the case where the exact solution of the Dirichlet problem admits corner singularities of type $r^{\alpha}, \alpha>0$, at a vertex of the computational domain $\Omega$ (where $r$ is the polar distance from the vertex). For the standard conforming $p$-version finite element method applied to the Poisson problems with corner singularities, it is well-known that the convergence rate is twice that predicted by standard a-priori bounds based on the regularity of the solution in Hilbertian Sobolev spaces [3]. Here, we show that a nearly order-doubling is also witnessed for $p$-IPDG method through an analytical and numerical study.

The rest of this work is organised as follows. Section 2 contains the model problem and definition of the interior penalty discontinuous Galerkin method. In Section 3, the question of suboptimal rate of convergence for the $p$-IPDG method is studied. In Section 4, some approximation bounds on the $p$-convergence of the $L^{2}$-projection operator are presented, which are subsequently utilized in Section 5 , where the convergence behaviour of $p$-IPDG on corner singularities is discussed. Section 6 contains some final comments.

\section{Preliminaries}

Let $\Omega$ be a bounded open polygonal domain in $\mathbb{R}^{2}$. We consider the Poisson problem

$$
-\Delta u=f \quad \text { in } \Omega
$$

with $f \in L^{2}(\Omega)$ together with Dirichlet boundary conditions

$$
u=g_{\mathrm{D}} \text { on } \partial \Omega
$$

The norm of $L^{2}(\omega)$, for $\omega \subset \Omega$ will be denoted by $\|\cdot\|_{L^{2}(\omega)}$. We shall also denote by $H^{s}(\omega)$ the standard Hilbertian Sobolev space of index $s \geq 0$ of real-valued functions defined on $\omega \subset \Omega$ and by $\|\cdot\|_{H^{s}(\omega)}$ it corresponding norm. We shall also refer in passing to the notions of an augmented Sobolev space (see [7] for the definition of augmented Sobolev spaces) and to function spaces constructed via the real method of interpolation (see, e.g., [14]).

Let $\mathcal{T}$ be a subdivision of the polygonal domain $\Omega$ into disjoint open elements $\kappa$ constructed via affine mappings $F_{\kappa}: \hat{\kappa} \rightarrow \kappa$ from some reference simplex or rectangle $\hat{\kappa}$, which are assumed to be constructed so as to ensure that the union of the closures of the elements $\kappa \in \mathcal{T}$ forms a covering of the closure of $\Omega$, i.e., $\bar{\Omega}=\cup_{\kappa \in \mathcal{T}} \bar{\kappa}$.

Definition 2.1 Let $\mathbf{p}:=\left(p_{\kappa}: \kappa \in \mathcal{T}\right)$ be the vector containing the polynomial degrees of the elements in a given subdivision $\mathcal{T}$ as described above. We define the finite element space $S^{\mathbf{p}}$ with respect to $\mathcal{T}$ and $\mathbf{p}$ by

$$
S^{\mathbf{p}}:=\left\{u \in L^{2}(\Omega):\left.u\right|_{\kappa} \circ F_{\kappa}^{-1} \in \mathcal{P}_{p_{\kappa}}(\hat{\kappa})\right\}
$$

where $\mathcal{P}_{p}(\hat{\kappa})$ is the space of polynomials of degree at most $p$ when $\hat{\kappa}$ is the reference simplex and of degree $p$ in each variable, when $\hat{\kappa}$ is the reference square.

We shall assume throughout that the mesh is fixed with a meshsize vector $\mathbf{h}:=\left(h_{\kappa}: \kappa \in \mathcal{T}\right)$ and the local polynomial degree vector $\mathbf{p}$, with $p_{\kappa} \geq 1$ for each $\kappa \in \mathcal{T}$, varies. For $\mathbf{p}$ we assume bounded local variation as $p_{\kappa} \rightarrow \infty$ for convergence, i.e., there exists constant $\rho \geq 1$, independent of $\mathbf{p}$, such that, for any pair of elements $\kappa$ and $\kappa^{\prime}$ in $\mathcal{T}$ which share a side, we have $\rho^{-1} \leq p_{\kappa} / p_{\kappa^{\prime}} \leq \rho$ as as $p_{\kappa} \rightarrow \infty$.

We denote by $\Gamma$ the union of all open one-dimensional element faces associated with the subdivision $\mathcal{T}$. We also assume that $\Gamma$ can be decomposed into two disjoint subsets $\partial \Omega$ and $\Gamma_{\text {int }}:=\Gamma \backslash \partial \Omega$. 
Further, we introduce some trace operators. Let $\kappa, \kappa^{\prime}$ be two (generic) elements sharing an interface $e \subset \Gamma_{\text {int }}$. Define the outward normal unit vectors $n^{+}$and $n^{-}$on $e$ corresponding to $\partial \kappa$ and $\partial \kappa^{\prime}$, respectively. Let $q \in S^{\mathbf{p}}$ and $\phi \in\left[S^{\mathbf{p}}\right]^{2}$. Then, with $q^{+}:=\left.q\right|_{\partial \kappa}, q^{-}:=\left.q\right|_{\partial \kappa^{\prime}}$ and $\phi^{+}:=\left.\phi\right|_{\partial \kappa}, \phi^{-}:=\left.\phi\right|_{\partial \kappa^{\prime}}$, we set

$$
\{q\}:=\frac{1}{2}\left(q^{+}+q^{-}\right),\{\phi\}:=\frac{1}{2}\left(\phi^{+}+\phi^{-}\right), \llbracket q \rrbracket:=q^{+} n^{+}+q^{-} n^{-}, \llbracket \phi \rrbracket:=\phi^{+} \cdot n^{+}+\phi^{-} \cdot n^{-} .
$$

If $e \subset \partial \Omega$, we define

$$
\{q\}:=q^{+},\{\phi\}:=\phi^{+}, \llbracket q \rrbracket:=q^{+} n, \llbracket \phi \rrbracket:=\phi^{+} \cdot n,
$$

where $n$ denotes the outward normal unit vector to $\partial \Omega$.

The discontinuous Galerkin finite element method for the problem (2.1), (2.2) reads:

$$
\text { Find } u_{\mathrm{DG}} \in S^{\mathbf{p}} \text { such that } B\left(u_{\mathrm{DG}}, v\right)=l(v) \quad \forall v \in S^{\mathbf{p}} \text {, }
$$

where

$$
B(u, v):=\sum_{\kappa \in \mathcal{T}} \int_{\kappa} \nabla u \cdot \nabla v \mathrm{~d} x+\int_{\Gamma}(\theta \llbracket u \rrbracket\{\nabla v\}-\{\nabla u\} \llbracket v \rrbracket+\sigma \llbracket u \rrbracket \llbracket v \rrbracket) \mathrm{d} s,
$$

and

$$
l(v):=\int_{\Omega} f v \mathrm{~d} x+\int_{\partial \Omega} g_{\mathrm{D}}(\theta \nabla v \cdot n+\sigma v) \mathrm{d} s,
$$

with $\theta \in\{-1,1\}$, and the function $\sigma$ to be defined later. If $\theta=-1$ we shall refer to the method as the symmetric version, and if $\theta=1$ we shall speak about (2.3) as the non-symmetric version of the IPDG method. In the simpler case when $\Gamma_{\text {int }}=\emptyset$ (i.e., we have a one-element mesh), we recover the $p$-version of the classical Nitsche's method for the imposition of non-homogeneous Dirichlet boundary conditions.

Related to the bilinear form, we define the (natural) DG-energy norm:

$$
\|w\|:=\left(\sum_{\kappa \in \mathcal{T}}\|\nabla w\|_{L^{2}(\kappa)}^{2}+\|\sqrt{\sigma} \llbracket w \rrbracket\|_{L^{2}(\Gamma)}^{2}\right)^{1 / 2}
$$

for any function $w \in L^{2}(\Omega)$ such that $\left.w\right|_{\kappa} \in H^{1}(\kappa)$ for all $\kappa \in \mathcal{T}$.

\section{The convergence of the $p$-IPDG method}

For the above DG method the following error bound holds, [11, 9, 7]:

Theorem 3.1 Let $\Omega$ be a polygonal domain, $\mathcal{T}$ a regular subdivision of $\Omega$ into shape-regular elements. We define

$$
\sigma:=C_{\sigma}\left\{\frac{\mathbf{p}^{2}}{\mathbf{h}}\right\}
$$

for some $C_{\sigma}>0$ (large enough) independent of $\mathbf{h}$ and of $\mathbf{p}$, but dependent on $\rho$ (which measures the local variation of the polynomial degree). If $u \in H^{1}(\Omega)$ is such that $\left.u\right|_{\kappa} \in H^{k_{\kappa}+1}(\kappa)$ for all $\kappa \in \mathcal{T}$ then the solution $u_{\mathrm{DG}} \in S^{\mathbf{P}}$ satisfies:

$$
\left\|u-u_{\mathrm{DG}} \mid\right\|^{2} \lesssim \sum_{\kappa \in \mathcal{T}} \frac{h_{\kappa}^{2 s_{\kappa}}}{p_{\kappa}^{2 k_{\kappa}-1}}\|u\|_{H^{k_{\kappa}+1}(\kappa)}^{2},
$$

with $1 \leq s_{\kappa} \leq \min \left\{p_{\kappa}, k_{\kappa}\right\}, p_{\kappa} \geq 1$.

(Here and in the remainder of this work $A \lesssim B$ and $A \gtrsim B$ is used instead of $A \leq C B$ and $A \geq C B$, respectively, for some positive generic constant $C$ independent of p.)

Hence, in particular, assuming a fixed mesh and a uniform polynomial degree $p_{\kappa}=p$, we conclude that for $u \in H^{k+1}(\Omega)$ and for $1 \leq s \leq \min \{p, k\}$, we have

$$
\left\|u-u_{\mathrm{DG}}\right\|\left\|\lesssim p^{-k+1 / 2}\right\| u \|_{H^{k+1}(\Omega)},
$$

i.e., the $p$-IPDG method (and its special case when $\Gamma_{\text {int }}=\emptyset$, the Nitsche's method) converge at a suboptimal rate with respect to the polynomial degree $p$, by half an order of $p$. 
In some cases it is possible to construct a non-trivial $H^{1}$-conforming subspace of the finite element space $S^{\mathbf{p}}$ up to the boundary with the same local polynomial degrees as $S^{\mathbf{p}}$, thereby facilitating the existence of an $H^{1}$-conforming interpolant [3, 12] of the analytical solution onto the finite element space, with $h p$-optimal convergence properties. Using this interpolant in the error analysis of the IPDG method, one can recover $h p$-optimal bounds (see, e.g., [6, Theorem 8.2] and the subsequent discussion therein). For instance, when the Dirichlet data $g_{\mathrm{D}}$ can be represented exactly as traces of finite element functions from $S^{\mathbf{p}}$ and the mesh contains simple hanging nodes (i.e., one hanging node per edge), a-priori error bounds of the form

$$
\left\|u-u_{\mathrm{DG}} \mid\right\| \lesssim p^{-k}\|u\|_{H^{k+1}(\Omega)},
$$

for $u \in H^{k+1}(\Omega)$, have been shown [6]. This provides motivation to seek the cause of the potential $p$-suboptimality in the general error bound (3.2) to boundary effects.

Remark 3.2 The hp-optimal rate of convergence for general Dirichlet boundary conditions can be recovered (when the mesh consists of quadrilateral elements), if we make additional regularity assumptions on the analytical solutions, namely, by assuming that it belongs element-wise to an augmented Sobolev space; we refer to [7] for more details.

Example 3.3 We consider the boundary-value problem

$$
-\Delta u=f \quad \text { in } \quad \Omega:=[-1,1] \times[0,2]
$$

with Dirichlet boundary conditions and $f$ such that

$$
u(x, y)=\left(x^{2}+y^{2}\right)^{\alpha / 2},
$$

for $\alpha \geq 1$ with $\alpha \notin 2 \mathbb{N}_{0}$. We approximate $u$ using the p-version IPDG method on a fixed regular mesh $\mathcal{T}$ (i.e., not containing any hanging nodes), which is constructed so that the origin $(0,0)$ is situated at the midpoint of the face of an element; we denote by $u_{\mathrm{DG}}$ the approximation of $u$ by the p-IPDG method.

The key point of the setup of Example 3.3 is that the singularity is not located at a vertex of mesh, and we have $u \in H^{\alpha+1-\epsilon}(\Omega)$, for all $\epsilon>0$. Therefore, the bound (3.2) implies

$$
\| u-u_{\mathrm{DG}}|| \lesssim p^{-\alpha+1 / 2+\epsilon}
$$

for all $\epsilon>0$, as $p \rightarrow \infty$. The sharpness of this bound is settled by the following result, concluding that the $p$-IPDG is indeed suboptimal in $p$ by half an order of $p$.

Proposition 3.4 For the Dirichlet problem described in Example 3.3 we have

$$
|| u-u_{\mathrm{DG}}|| \gtrsim p^{-\alpha+1 / 2}
$$

as $p \rightarrow \infty$.

Proof We denote by

$$
E_{p}(v):=\inf _{v_{p} \in \mathcal{P}_{p}([-1,1])}\left\|v-v_{p}\right\|_{L^{2}(-1,1)}
$$

i.e., the best approximation in the $L^{2}$-norm of a function $v \in L^{2}([-1,1])$ by univariate polynomials of degree $p$ on the interval $[-1,1]$. Theorem 9 of [8] implies that for functions of the form $v=|x|^{\alpha}$ for $\alpha>0$ and $\alpha \notin 2 \mathbb{N}_{0}$, we have

$$
E_{p}(v) \gtrsim p^{-\alpha-1 / 2}
$$

for some constant $C>0$. Moreover, it is a straightforward matter to see (cf. [8, Lemma 2]) that a rescaling of the domain from $[-1,1]$ to $[-h / 2, h / 2]$, for some $h>0$ has only the effect of altering the constant in (3.4) (since we have assumed a fixed mesh).

Let $\kappa$ be the element whose boundary contains the origin $(0,0)$. We consider the part of the boundary $\partial \kappa_{1}:=\left[-h_{\kappa} / 2, h_{\kappa} / 2\right] \times\{0\}$ on which we have $u(x, 0)=|x|^{\alpha}$. The bound (3.4) then implies that in this case we have

$$
\left\|u-u_{\mathrm{DG}}\right\|_{L^{2}\left(\partial \kappa_{1}\right)} \gtrsim p^{-\alpha-1 / 2}
$$


for some constant $C>0$ independent of $p$. Therefore, recalling the definition of $\sigma$, we conclude that

$$
\left\|u-u_{\mathrm{DG}}\right\|^{2}=\sum_{\kappa \in \mathcal{T}}\left\|\nabla\left(u-u_{\mathrm{DG}}\right)\right\|_{L^{2}(\kappa)}^{2}+\left\|\sqrt{\sigma}\left(u-u_{\mathrm{DG}}\right)\right\|_{L^{2}\left(\Gamma \backslash \partial \kappa_{1}\right)}^{2}+\left\|\sqrt{\sigma}\left(u-u_{\mathrm{DG}}\right)\right\|_{L^{2}\left(\partial \kappa_{1}\right)}^{2} \gtrsim p^{-2 \alpha+1} .
$$

To investigate the setting of Example 3.3 numerically, we consider $p$-IPDG with one element (i.e., $\left.\Gamma_{\text {int }}=\emptyset\right)$; the $p$-convergence history is shown in Figure 1. The error appears to oscillate in magnitude for even and for odd $p$. One possible reason behind this is the symmetry of the analytical solution (which is an even function); for low polynomial degrees $p$ we observe that the even degree approximations are more accurate than the approximations with odd polynomial degree basis functions. We point out that quadruple precision arithmetic has been used in the numerical experiments of this work, along with a geometrically graded composite quadrature rules, graded towards the point $(0,0)$.

In Table 1, the error in the DG-norm is presented, grouped in even and odd polynomial degree approximations, along with the $p$-convergence rates $r(p)$ calculated as follows:

$$
r(p)=-\frac{\log \operatorname{error}(p)-\log \operatorname{error}(p-2)}{\log p-\log (p-2)},
$$

with $p=4,6,8, \ldots$ or $p=3,5,7, \ldots$, respectively, where $\operatorname{error}(p)$ denotes the approximation error in the respective (semi)norm when polynomial degree $p$ basis functions are used.

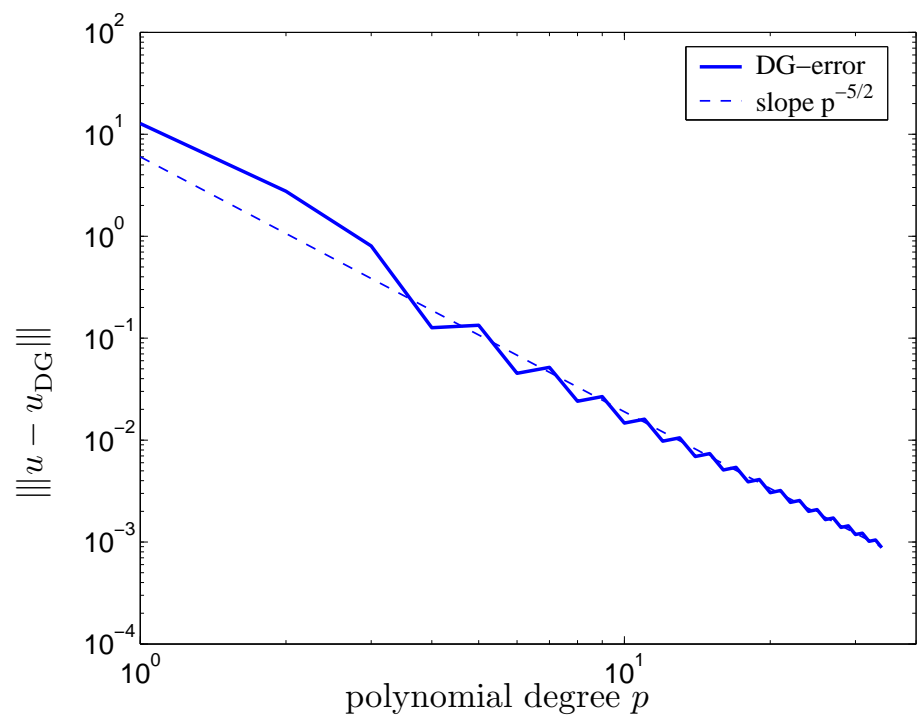

Figure 1: Example 3.3: Convergence history for the $p$-IPDG for $\alpha=3$.

Next, we investigate the $p$-convergence of the individual components of the DG-norm error. To this end, recalling that we work on a single-element mesh (and, therefore, $\Omega=\kappa$ and $\Gamma=\partial \kappa$ ), we study the $p$-convergence of the errors

$$
\left\|\nabla\left(u-u_{\mathrm{DG}}\right)\right\|_{L^{2}(\Omega)} \quad \text { and } \quad\left\|\sqrt{\sigma}\left(u-u_{\mathrm{DG}}\right)\right\|_{L^{2}(\partial \Omega)}
$$

as $p \rightarrow \infty$. The errors and the corresponding $p$-convergence rates are given in Tables 2 and 3 , where the dominance of the term $\left\|\sqrt{\sigma}\left(u-u_{\mathrm{DG}}\right)\right\|_{L^{2}(\partial \Omega)}$ over the term $\left\|\nabla\left(u-u_{\mathrm{DG}}\right)\right\|_{L^{2}(\Omega)}$ is manifested; indeed the $p$-convergence behaviour of $\left\|\sqrt{\sigma}\left(u-u_{\mathrm{DG}}\right)\right\|_{L^{2}(\partial \Omega)}$ essentially determines the $p$-convergence of the DGnorm error (cf. Table 1). We note that for odd $p$ the convergence rate appears to be increasing towards the value $5 / 2$.

\section{Properties of the $L^{2}$-projection operator}

In the present section, we refine the analysis of [9] of the properties of the $L^{2}$-projection operator. We start with an improvement of the one-dimensional result [9, Lemma 3.5]. 


\begin{tabular}{|c||c|c||c||c|c|}
\hline$p$ & $\left\|u-u_{\mathrm{DG}}\right\|$ & $r(p)$ & $p$ & $\left\|u-u_{\mathrm{DG}}\right\|$ & $r(p)$ \\
\hline \hline 2 & $2.7634+00$ & - & 1 & $1.2728+01$ & - \\
\hline 4 & $1.2643-01$ & 4.45 & 3 & $8.0330-01$ & 2.51 \\
\hline 6 & $4.5238-02$ & 2.53 & 5 & $1.3381-01$ & 3.51 \\
\hline 8 & $2.4020-02$ & 2.20 & 7 & $5.1617-02$ & 2.83 \\
\hline 10 & $1.4674-02$ & 2.21 & 9 & $2.6717-02$ & 2.62 \\
\hline 12 & $9.7691-03$ & 2.23 & 11 & $1.6029-02$ & 2.55 \\
\hline 14 & $6.9029-03$ & 2.25 & 13 & $1.0535-02$ & 2.51 \\
\hline 16 & $5.0964-03$ & 2.27 & 15 & $7.3725-03$ & 2.50 \\
\hline 18 & $3.8919-03$ & 2.29 & 17 & $5.4024-03$ & 2.48 \\
\hline 20 & $3.0529-03$ & 2.30 & 19 & $4.1008-03$ & 2.48 \\
\hline 22 & $2.4478-03$ & 2.32 & 21 & $3.2011-03$ & 2.47 \\
\hline 24 & $1.9987-03$ & 2.33 & 23 & $2.5562-03$ & 2.47 \\
\hline 26 & $1.6573-03$ & 2.34 & 25 & $2.0801-03$ & 2.47 \\
\hline 28 & $1.3925-03$ & 2.35 & 27 & $1.7198-03$ & 2.47 \\
\hline 30 & $1.1835-03$ & 2.36 & 29 & $1.4414-03$ & 2.47 \\
\hline 32 & $1.0160-03$ & 2.36 & 31 & $1.2223-03$ & 2.47 \\
\hline 34 & $8.7996-04$ & 2.37 & 33 & $1.0473-03$ & 2.47 \\
\hline
\end{tabular}

Table 1: Example 3.3: DG-norm errors and convergence rates for the $p$-IPDG for $\alpha=3$.

\begin{tabular}{|c||c|c||c||c|c|}
\hline$p$ & $\left\|\nabla\left(u-u_{\mathrm{DG}}\right)\right\|_{L^{2}(\Omega)}$ & $r(p)$ & $p$ & $\left\|\nabla\left(u-u_{\mathrm{DG}}\right)\right\|_{L^{2}(\Omega)}$ & $r(p)$ \\
\hline \hline 2 & $1.7896+00$ & - & 1 & $8.7949+00$ & - \\
\hline 4 & $5.3593-02$ & 5.06 & 3 & $4.4791-01$ & 2.71 \\
\hline 6 & $1.5150-02$ & 3.12 & 5 & $4.8958-02$ & 4.33 \\
\hline 8 & $6.5414-03$ & 2.92 & 7 & $1.4964-02$ & 3.52 \\
\hline 10 & $3.4303-03$ & 2.89 & 9 & $6.5336-03$ & 3.30 \\
\hline 12 & $2.0212-03$ & 2.90 & 11 & $3.4306-03$ & 3.21 \\
\hline 14 & $1.2898-03$ & 2.91 & 13 & $2.0217-03$ & 3.17 \\
\hline 16 & $8.7268-04$ & 2.93 & 15 & $1.2901-03$ & 3.14 \\
\hline 18 & $6.1753-04$ & 2.94 & 17 & $8.7284-04$ & 3.12 \\
\hline 20 & $4.5281-04$ & 2.94 & 19 & $6.1763-04$ & 3.11 \\
\hline 22 & $3.4175-04$ & 2.95 & 21 & $4.5287-04$ & 3.10 \\
\hline 24 & $2.6419-04$ & 2.96 & 23 & $3.4179-04$ & 3.09 \\
\hline 26 & $2.0839-04$ & 2.96 & 25 & $2.6422-04$ & 3.09 \\
\hline 28 & $1.6724-04$ & 2.97 & 27 & $2.0841-04$ & 3.09 \\
\hline 30 & $1.3623-04$ & 2.97 & 29 & $1.6726-04$ & 3.08 \\
\hline 32 & $1.1243-04$ & 2.98 & 31 & $1.3624-04$ & 3.08 \\
\hline 34 & $9.3853-05$ & 2.98 & 33 & $1.1244-04$ & 3.07 \\
\hline
\end{tabular}

Table 2: Example 3.3: $\left\|\nabla\left(u-u_{\mathrm{DG}}\right)\right\|_{L^{2}(\Omega)}$-error and convergence rates for $\alpha=3$. 


\begin{tabular}{|c||c|c||c||c|c|}
\hline$p$ & $\left\|\sqrt{\sigma}\left(u-u_{\mathrm{DG}}\right)\right\|_{L^{2}(\partial \Omega)}$ & $r(p)$ & $p$ & $\left\|\sqrt{\sigma}\left(u-u_{\mathrm{DG}}\right)\right\|_{L^{2}(\partial \Omega)}$ & $r(p)$ \\
\hline \hline 2 & $2.1056+00$ & - & 1 & $9.2003+00$ & - \\
\hline 4 & $1.1451-01$ & 4.20 & 3 & $6.6684-01$ & 2.39 \\
\hline 6 & $4.2626-02$ & 2.44 & 5 & $1.2454-01$ & 3.28 \\
\hline 8 & $2.3113-02$ & 2.13 & 7 & $4.9401-02$ & 2.75 \\
\hline 10 & $1.4267-02$ & 2.16 & 9 & $2.5906-02$ & 2.57 \\
\hline 12 & $9.5577-03$ & 2.20 & 11 & $1.5657-02$ & 2.50 \\
\hline 14 & $6.7814-03$ & 2.23 & 13 & $1.0339-02$ & 2.48 \\
\hline 16 & $5.0211-03$ & 2.25 & 15 & $7.2588-03$ & 2.47 \\
\hline 18 & $3.8426-03$ & 2.27 & 17 & $5.3314-03$ & 2.47 \\
\hline 20 & $3.0191-03$ & 2.29 & 19 & $4.0541-03$ & 2.46 \\
\hline 22 & $2.4238-03$ & 2.30 & 21 & $3.1689-03$ & 2.46 \\
\hline 24 & $1.9812-03$ & 2.32 & 23 & $2.5333-03$ & 2.46 \\
\hline 26 & $1.6442-03$ & 2.33 & 25 & $2.0633-03$ & 2.46 \\
\hline 28 & $1.3825-03$ & 2.34 & 27 & $1.7071-03$ & 2.46 \\
\hline 30 & $1.1757-03$ & 2.35 & 29 & $1.4316-03$ & 2.46 \\
\hline 32 & $1.0098-03$ & 2.36 & 31 & $1.2147-03$ & 2.46 \\
\hline 34 & $8.7494-03$ & 2.36 & 33 & $1.0412-03$ & 2.46 \\
\hline
\end{tabular}

Table 3: Example 3.3: $\left\|\sqrt{\sigma}\left(u-u_{\mathrm{DG}}\right)\right\|_{L^{2}(\partial \Omega)}$-error and convergence rates for $\alpha=3$.

Lemma 4.1 Let $I=(-1,1)$ and denote $\Pi_{p}: L^{2}(I) \rightarrow \mathcal{P}_{p}$ the $L^{2}$-projection operator. Then

$$
\left|\left(\Pi_{p} u\right)( \pm 1)\right| \lesssim\|u\|_{L^{2}(I)}^{1 / 2}\|u\|_{H^{1}(I)}^{1 / 2} \quad \forall u \in H^{1}(I) .
$$

In particular, therefore,

$$
\left|\left(\Pi_{p} u\right)( \pm 1)\right| \lesssim\|u\|_{B_{2,1}^{1 / 2}(I)} \quad \forall u \in B_{2,1}^{1 / 2}(I) .
$$

Here, the space $B_{2,1}^{1 / 2}(I)=\left(L^{2}(I), H^{1}(I)\right)_{1 / 2,1}$ is the interpolation space obtained by the real method (see, e.g., [14]).

Proof We only show the multiplicative inequality (4.1) since the bound (4.2) follows from [14, Lemma 25.3]. Also, we will only consider the case $p \geq 2$ and restrict out attention to evaluation at the right endpoint +1 . Since $\|u\|_{L^{\infty}(I)}^{2} \lesssim\|u\|_{L^{2}(I)}\|u\|_{H^{1}(I)}$, it suffices to establish the inequality for $u-\Pi_{p} u$. Following $[9$, Lemma 3.5], we expand $u$ and $u^{\prime}$ in Legendre series:

$$
\begin{aligned}
u=\sum_{i=0}^{\infty} u_{i} L_{i}, & u_{i}=\frac{2 i+1}{2} \int_{I} u(x) \mathrm{d} x, \\
u^{\prime}=\sum_{i=0}^{\infty} b_{i} L_{i}, & b_{i}=\frac{2 i+1}{2} \int_{I} u^{\prime}(x) \mathrm{d} x .
\end{aligned}
$$

Orthogonality properties of the Legendre polynomials $L_{i}$ imply (see the proof of [9, Lemma 3.5] for details)

$$
u_{i}=\frac{b_{i-1}}{2 i-1}-\frac{b_{i+1}}{2 i+3}, \quad i \geq 2 .
$$

Since $L_{i}(1)=1$ for all $i \in \mathbb{N}_{0}$, we get

$$
\left(u-\Pi_{p} u\right)(1)=\sum_{i=p+1}^{\infty} u_{i}=\frac{b_{p}}{2 p+1}+\frac{b_{p+1}}{2 p+3},
$$

and therefore

$$
\left|\left(u-\Pi_{p} u\right)(1)\right|^{2}=\left|\sum_{i=p+1}^{\infty} \hat{u}_{i}\right|^{2} \leq 2\left(\frac{b_{p}}{2 p+1}\right)^{2}+2\left(\frac{b_{p+1}}{2 p+3}\right)^{2} .
$$


The terms in the last expression are now estimated using a telescoping sum:

$$
\begin{aligned}
\left(\frac{b_{p}}{2 p+1}\right)^{2} & =\sum_{r=p}^{\infty}\left(\frac{b_{r}}{2 r+1}\right)^{2}-\left(\frac{b_{r+2}}{2(r+2)+1}\right)^{2} \\
& =\sum_{r=p}^{\infty}\left(\frac{b_{r}}{2 r+1}-\frac{b_{r+2}}{2(r+2)+1}\right)\left(\frac{b_{r}}{2 r+1}+\frac{b_{r+2}}{2(r+2)+1}\right) \\
& =\sum_{r=p}^{\infty} u_{r+1}\left(\frac{b_{r}}{2 r+1}+\frac{b_{r+2}}{2(r+2)+1}\right) \\
& =\sum_{r=p}^{\infty} u_{r+1} \frac{1}{\sqrt{2(r+1)+1}} \sqrt{2(r+1)+1}\left(\frac{b_{r}}{2 r+1}+\frac{b_{r+2}}{2(r+2)+1}\right) \\
& \lesssim\left(\sum_{r=p}^{\infty} \frac{1}{2(r+1)+1}\left|u_{r}\right|^{2}\right)^{1 / 2}\left(\sum_{r=p}^{\infty}(2 r+1)\left(\frac{b_{r}}{2 r+1}\right)^{2}\right)^{1 / 2} \\
& \lesssim\|u\|_{L^{2}(I)}\left\|u^{\prime}\right\|_{L^{2}(I)},
\end{aligned}
$$

where we have used $\|u\|_{L^{2}(I)}^{2}=\sum_{i=0}^{\infty}\left|u_{i}\right|^{2} \frac{2}{2 i+1}$ and $\left\|u^{\prime}\right\|_{L^{2}(I)}^{2}=\sum_{i=0}^{\infty}\left|b_{i}\right|^{2} \frac{2}{2 i+1}$. We therefore conclude $\left|\left(u-\Pi_{p} u\right)(1)\right|^{2} \lesssim\|u\|_{L^{2}}\left\|u^{\prime}\right\|_{L^{2}}$. as desired.

Tensorization of Lemma 4.1 gives a result for the square.

Lemma 4.2 Let $I=(-1,1)$ and $S=I^{2}$. Denote by $\Pi_{p}: L^{2}(S) \rightarrow \mathcal{P}_{p}$ the $L^{2}(S)$-projection. Then:

$$
\left\|\Pi_{p} u\right\|_{L^{2}(\partial S)}^{2} \lesssim\|u\|_{L^{2}(S)}\|u\|_{H^{1}(S)} \quad \forall u \in H^{1}(S) .
$$

This implies

$$
\left\|\Pi_{p} u\right\|_{L^{2}(\partial S)} \lesssim\|u\|_{B_{2,1}^{1 / 2}(S)} \quad \forall u \in B_{2,1}^{1 / 2}(S) .
$$

Proof The two-dimensional $L^{2}$-projection is the tensor product of one-dimensional projection operators: $\Pi_{p}=\Pi_{p}^{x} \circ \Pi_{p}^{y}$. Then, letting $\Gamma=I \times\{1\}$ be one edge of $S$ :

$$
\begin{aligned}
\left\|\Pi_{p} u\right\|_{L^{2}(\Gamma)}^{2} & =\int_{x \in I}\left|\Pi_{p}^{y}\left(\Pi_{p}^{x} u\right)(x, 1)\right|^{2} \mathrm{~d} x \lesssim \int_{x \in I}\left\|\Pi_{p}^{x} u(x, \cdot)\right\|_{L^{2}(I)}\left(\left\|\Pi_{p}^{x} u(x, \cdot)\right\|_{L^{2}(I)}+\left\|\partial_{y} \Pi_{p}^{x} u(x, \cdot)\right\|_{L^{2}(I)}\right) \mathrm{d} x \\
& \lesssim\|u\|_{L^{2}(S)}^{2}+\int_{x \in I}\left\|\Pi_{p}^{x} u(x, \cdot)\right\|_{L^{2}(I)}\left\|\Pi_{p}^{x} \partial_{y} u(x, \cdot)\right\|_{L^{2}(I)} \mathrm{d} x
\end{aligned}
$$

For any $t>0$, we can estimate further

$$
\begin{aligned}
\int_{x \in I}\left\|\Pi_{p}^{x} u(x, \cdot)\right\|_{L^{2}(I)}\left\|\Pi_{p}^{x} \partial_{y} u(x, \cdot)\right\|_{L^{2}(I)} \mathrm{d} x & \lesssim \int_{x \in I} t^{-1}\left\|\Pi_{p}^{x} u(x, \cdot)\right\|_{L^{2}(I)}^{2}+t\left\|\Pi_{p}^{x} \partial_{y} u(x, \cdot)\right\|_{L^{2}(I)}^{2} \mathrm{~d} x \\
& \lesssim t^{-1}\|u\|_{L^{2}(S)}^{2}+t\|\nabla u\|_{L^{2}(S)}^{2}
\end{aligned}
$$

Optimizing $t$ gives

$$
\int_{x \in I}\left\|\Pi_{p}^{x} u(x, \cdot)\right\|_{L^{2}(I)}\left\|\Pi_{p}^{x} \partial_{y} u(x, \cdot)\right\|_{L^{2}(I)} \lesssim\|u\|_{L^{2}(S)}\|u\|_{H^{1}(S)}
$$

Remark 4.3 1. Lemma 4.1 together with standard polynomial approximation properties implies for every $k>1 / 2$ :

$$
\left|\left(u-\Pi_{p} u\right)( \pm 1)\right| \leq C_{k} p^{-(k-1 / 2)}\|u\|_{H^{k}(-1,1)} \quad \forall u \in H^{k}(-1,1),
$$

for some constant $C_{k}>0$, depending on the Sobolev index $k$. 
2. Lemma 4.2 can be generalized to hypercubes in $\mathbb{R}^{d}, d \geq 2$.

We conclude this section with a statement about the approximation properties of the $L^{2}$-projector on squares.

Lemma 4.4 Let $S=(-1,1)^{2}$ and denote by $\Pi_{p}: L^{2}(S) \rightarrow \mathcal{P}_{p}$ the $L^{2}(S)$-projection. Then for all $u \in H^{1}(S)$ :

$$
\begin{aligned}
\left\|u-\Pi_{p} u\right\|_{H^{1}(S)} & \lesssim \sqrt{p} \inf _{q \in \mathcal{P}_{p}}\|u-q\|_{H^{1}(S)} \\
\left\|u-\Pi_{p} u\right\|_{L^{2}(\partial S)} & \lesssim \inf _{q \in \mathcal{P}_{p}}\|u-q\|_{B_{2,1}^{1 / 2}(S)} \lesssim \inf _{q \in \mathcal{P}_{p}}\|u-q\|_{L^{2}(S)}^{1 / 2}\|u-q\|_{H^{1}(S)}^{1 / 2}
\end{aligned}
$$

Proof The first estimate follows immediately from [4, Thm. 2.4], which states $\left\|\Pi_{p} u\right\|_{H^{1}(S)} \lesssim \sqrt{p}\|u\|_{H^{1}(S)}$ for all $u \in H^{1}(S)$. The second bound follows from Lemma 4.2.

\section{Convergence in the presence of corner singularities}

When the exact solution $u$ admits a corner singularity of type $r^{\alpha}$ (here $(r, \theta)$ denote polar coordinates and the origin is assumed to be a vertex of $\Omega$ ), it is known [3] that the conforming p-version finite element method applied to the problem (2.1), (2.2) obeys a bound of the form

$$
\left\|\nabla\left(u-u_{p, \operatorname{con} f}\right)\right\| \lesssim p^{-2 \alpha}
$$

where $u_{p, \text { conf }}$ denotes the conforming $p$-version finite element approximation. This is the well-known order-doubling phenomenon of the $p$-version finite element method in the presence of corner singularities. (Note that $u=r^{\alpha} \in H^{\alpha+1-\epsilon}(\Omega)$ for all $\epsilon>0$.)

In order to investigate the convergence behaviour of the $p$-IPDG method for problems with corner singularities, we consider the following example.

Example 5.1 We consider the boundary-value problem

$$
-\Delta u=f \quad \text { in } \quad \Omega:=[0,1] \times[0,1]
$$

with Dirichlet boundary conditions and $f$ such that

$$
u(x, y)=\left(x^{2}+y^{2}\right)^{\alpha / 2} .
$$

We approximate $u$ using the p-IPDG method on a fixed Cartesian mesh $\mathcal{T}$, denoting the p-IPDG solution by $u_{\mathrm{DG}}$.

Before formulating the convergence result for the $p$-IPDG applied to Example 5.1, we state an approximation result:

Lemma 5.2 Let $S=(0,1)^{2}$ and a function $u \in H^{1}(S)$ be given of the form $u(r, \theta)=r^{\alpha} \phi(\theta)$, where $(r, \theta)$ are polar coordinates with respect to the origin. The function $\phi$ is assumed to be smooth. Let $\Pi_{p}^{H^{1}}$ and $\Pi_{p}^{L^{2}}$ denote the $H^{1}(S)$ and $L^{2}(S)$-projection operators onto $\mathcal{P}_{p}$. Then:

$$
\begin{aligned}
\left\|u-\Pi_{p}^{H^{1}} u\right\|_{H^{1}(S)}+p\left\|u-\Pi_{p}^{H^{1}} u\right\|_{L^{2}(S)}+p^{1 / 2}\left\|u-\Pi_{p}^{H^{1}} u\right\|_{L^{2}(\partial S)} & \lesssim p^{-2 \alpha} \\
p^{-1 / 2}\left\|u-\Pi_{p}^{L^{2}} u\right\|_{H^{1}(S)}+p^{2}\left\|u-\Pi_{p}^{L^{2}} u\right\|_{L^{2}(S)}+p^{3 / 4}\left\|u-\Pi_{p}^{L^{2}} u\right\|_{L^{2}(\partial S)} & \lesssim p^{-2 \alpha} .
\end{aligned}
$$

Proof By [2, Theorem 2.6], we have $\left\|u-\Pi_{p}^{H^{1}} u\right\|_{H^{1}(S)} \lesssim p^{-2 \alpha}$. A standard duality argument for the convex domain $S=(0,1)^{2}$ then gives additionally $\left\|u-\Pi_{p}^{H^{1}} u\right\|_{L^{2}(S)} \lesssim p^{-2 \alpha-1}$. The estimate for $\| u-$ $\Pi_{p}^{H^{1}} u \|_{L^{2}(\partial S)}$ finally follows from the multiplicative trace inequality.

The $H^{1}$-estimate in (5.2) follows from (5.1) and Lemma 4.4. The $L^{2}$-bound follows from [2, Theorems $3.10,3.12]$. These two estimates together with Lemma 4.4 imply the $L^{2}(\partial S)$-bound. 
Proposition 5.3 Let $\alpha>2$. Then the p-IPDG for the problem described in Example 5.1, obeys the bound

$$
|| u-u_{\mathrm{DG}}|| \lesssim p^{-2 \alpha+1 / 2},
$$

as $p \rightarrow \infty$.

Proof For simplicity of presentation, we consider the case of a one-element mesh, i.e., $\mathcal{T}=\{\Omega\}$; the general case follows analogously. We consider the extension $\tilde{B}$ of the bilinear form $(2.4)$ into $\left(H^{1}(\Omega)+\right.$ $\left.S^{\mathbf{p}}\right) \times\left(H^{1}(\Omega)+S^{\mathbf{p}}\right)$ defined by

$$
\tilde{B}(u, v):=\int_{\Omega} \nabla u \cdot \nabla v \mathrm{~d} x+\int_{\partial \Omega}\left(\theta u \Pi_{p}(\nabla v) \cdot n-\Pi_{p}(\nabla u) \cdot n v+\sigma u v\right) \mathrm{d} s,
$$

where $\Pi_{p}: L^{2}(\Omega) \rightarrow\left[S^{\mathbf{p}}\right]^{2}$ here denotes the (component-wise) $L^{2}$-projection operator onto $\left[S^{\mathbf{p}}\right]^{2}$. Note that $\tilde{B}=B$ on $S^{\mathbf{p}} \times S^{\mathbf{p}}$. Also $\tilde{B}$ is coercive and continuous with respect to the energy norm (these properties can be verified using standard arguments). Therefore, Strang's Second Lemma implies

$$
\left\|u-u_{\mathrm{DG}}\left|\left\|\lesssim \inf _{v \in S^{\mathbf{p}}}|\|u-v\||+\sup _{\chi \in S^{\mathbf{P}} \backslash\{0\}} \frac{|R(u, \chi)|}{\|\chi\| \mid},\right.\right.\right.
$$

where the residual $R(u, \chi):=B(u, \chi)-\tilde{B}(u, \chi)$ equals

$$
R(u, \chi)=\int_{\partial \Omega}\left(\nabla u \cdot n-\Pi_{p}(\nabla u) \cdot n\right) \chi \mathrm{d} s .
$$

Since the components of $\nabla u$ of have the form $r^{\alpha-1} \widetilde{\phi}(\theta)$ for a smooth $\widetilde{\phi}$, we get from Lemma 5.2

$$
\begin{aligned}
& \sup _{\chi \in S^{P} \backslash\{0\}} \frac{|R(u, \chi)|}{\|\| \chi \|} \leq\left\|\sigma^{-1 / 2}\left(\nabla u \cdot n-\Pi_{p}(\nabla u) \cdot n\right)\right\|_{L^{2}(\partial \Omega)} \lesssim p^{-1}\left\|\nabla u-\Pi_{p}(\nabla u)\right\|_{L^{2}(\partial \Omega)} \\
& \lesssim p^{-1} p^{-2(\alpha-1)-3 / 4}=p^{-2 \alpha+1 / 4} .
\end{aligned}
$$

Additionally, Lemma 5.2 implies

$$
\inf _{q \in S^{\mathbf{p}}} \mid\|u-q\| \| p^{-2 \alpha+1 / 2}
$$

which allows us to conclude the proof.

To investigate the convergence history numerically, we consider the $p$-IPDG with one element (i.e., $\left.\Gamma_{\text {int }}=\emptyset\right)$; the errors in the DG-norm along with the corresponding convergence rates calculated in this case by the formula

$$
r(p)=-\frac{\log \text { error }(p)-\log \text { error }(p-1)}{\log p-\log (p-1)},
$$

are given in Table 4. The convergence rate appears to be higher than what the bound (5.3) suggests for this case (i.e., rate 11/2), but lower than 6 , which is the expected convergence rate for the conforming $p$-version finite element method for the same problem. It is not known at this point, if the bound (5.3) is sharp, or if it can be further improved.

\section{Conclusions}

From the discussion above, we conclude that error bounds of the form (3.1) are sharp and that the $p$ suboptimality is a result of suboptimal approximation of Dirichlet boundary conditions (assuming that the underlying mesh is reasonable, e.g., containing simple hanging nodes [12]). Hence, in situations where the Dirichlet boundary conditions are represented exactly (or approximated super-optimally) by traces of finite element functions, the $p$-IPDG method converges optimally. We also investigated the question of convergence rates for the $p$-IPDG method for problems with corner singularities, finding that the convergence rate of the $p$-IPDG method for such problems appears to be slightly suboptimal. 


\begin{tabular}{|c||c|c||c||c|c|}
\hline$p$ & ||$\left|u-u_{\text {DG }}\right| \mid$ & $r(p)$ & $p$ & $\left|\| u-u_{\text {DG }}\right| \mid$ & $r(p)$ \\
\hline \hline 1 & $1.5850+01$ & - & 18 & $4.4933-06$ & 5.84 \\
\hline 2 & $3.4096+00$ & 2.21 & 19 & $3.2758-06$ & 5.84 \\
\hline 3 & $2.2218-01$ & 6.73 & 20 & $2.4270-06$ & 5.84 \\
\hline 4 & $3.9386-02$ & 6.01 & 21 & $1.8243-06$ & 5.85 \\
\hline 5 & $8.7734-03$ & 6.72 & 22 & $1.3895-06$ & 5.85 \\
\hline 6 & $2.8793-03$ & 6.11 & 23 & $1.0710-06$ & 5.85 \\
\hline 7 & $1.1399-03$ & 6.01 & 24 & $8.3472-07$ & 5.85 \\
\hline 8 & $5.1657-04$ & 5.92 & 25 & $6.5718-07$ & 5.85 \\
\hline 9 & $2.5833-04$ & 5.88 & 26 & $5.2211-07$ & 5.86 \\
\hline 10 & $1.3929-04$ & 5.86 & 27 & $4.1855-07$ & 5.85 \\
\hline 11 & $7.9756-05$ & 5.85 & 28 & $3.3817-07$ & 5.86 \\
\hline 12 & $4.7967-05$ & 5.84 & 29 & $2.7512-07$ & 5.87 \\
\hline 13 & $3.0056-05$ & 5.84 & 30 & $2.2542-07$ & 5.87 \\
\hline 14 & $1.9499-05$ & 5.83 & 31 & $1.8602-07$ & 5.85 \\
\hline 15 & $1.3033-05$ & 5.83 & 32 & $1.5429-07$ & 5.89 \\
\hline 16 & $8.9413-06$ & 5.83 & 33 & $1.2914-07$ & 5.78 \\
\hline 17 & $6.2750-06$ & 5.84 & 34 & $1.0853-07$ & 5.82 \\
\hline
\end{tabular}

Table 4: Example 5.1: $p$-Convergence of $\| u-u_{\mathrm{DG}}|| \mid$ for $\alpha=3$.

\section{References}

[1] BABUŠKA, I. The finite element method with penalty. Math. Comput. 27 (1973), 221-228.

[2] Babuska, I., And Guo, B. Direct and inverse approximation theorems for the $p$-version of the finite element method in the framework of weighted Besov spaces. I: Approximability of functions in the weighted Besov spaces. SIAM J. Numer. Anal. 39, 5 (2001), 1512-1538.

[3] BABUŠKa, I., AND SURI, M. The optimal convergence rate of the $p$-version of the finite element method. SIAM J. Numer. Anal. 24, 4 (1987), 750-776.

[4] Canuto, C., and Quarteroni, A. Approximation results for orthogonal polynomials in Sobolev spaces. Math. Comput. 38, 257 (1982), 67-86.

[5] Cockburn, B., Karniadakis, G. E., and Shu, C.-W. The development of discontinuous Galerkin methods. In Discontinuous Galerkin methods (Newport, RI, 1999), vol. 11 of Lect. Notes Comput. Sci. Eng. Springer, Berlin, 2000, pp. 3-50.

[6] Georgoulis, E. $h p$-version interior penalty discontinuous Galerkin finite element methods on anisotropic meshes. Int. J. Numer. Anal. Model. 3 (2006), 52-79.

[7] Georgoulis, E. H., And Süli, E. Optimal error estimates for the $h p$-version interior penalty discontinuous Galerkin finite element method. IMA J. Numer. Anal. 25, 1 (2005), 205-220.

[8] Gui, W., And BabušKa, I. The $h, p$ and $h-p$ versions of the finite element method in 1 dimension. I. The error analysis of the $p$-version. Numer. Math. 49, 6 (1986), 577-612.

[9] Houston, P., Schwab, C., And Süli, E. Discontinuous $h p$-finite element methods for advectiondiffusion-reaction problems. SIAM J. Numer. Anal. 39, 6 (2002), 2133-2163 (electronic).

[10] Nitsche, J. Über ein Variationsprinzip zur Lösung von Dirichlet-Problemen bei Verwendung von Teilräumen, die keinen Randbedingungen unterworfen sind. Abh. Math. Sem. Univ. Hamburg 36 (1971), 9-15. Collection of articles dedicated to Lothar Collatz on his sixtieth birthday.

[11] Rivière, B., Wheeler, M. F., And Girault, V. A priori error estimates for finite element methods based on discontinuous approximation spaces for elliptic problems. SIAM J. Numer. Anal. 39, 3 (2001), 902-931 (electronic). 
[12] Schwab, C. p- and hp-finite element methods. Numerical Mathematics and Scientific Computation. The Clarendon Press Oxford University Press, New York, 1998. Theory and applications in solid and fluid mechanics.

[13] Stamm, B., and Winler, T. P. hp-Optimal discontinuous Galerkin methods for linear elliptic problems. Tech. rep., 2007. EPFL/IACS report 07.2007.

[14] Tartar, L. An introduction to Sobolev spaces and interpolation spaces, vol. 3 of Lecture Notes of the Unione Matematica Italiana. Springer, Berlin, 2007. 